\title{
The use of pigmentation and shading information in recognising the sex and identities of faces
}

\author{
Vicki Bruce \\ Department of Psychology, University of Stirling, Stirling, FK9 4LA, UK \\ Steve Langton \\ Department of Psychology, University of Nottingham, Nottingham, NG7 2RD, UK \\ Received 5 April 1993, in revised form 23 March 1994
}

\begin{abstract}
An investigation of what can be learned about representational processes in face recognition from the independent and combined effects of inverting and negating facial images is reported. In experiment 1 , independent effects of inversion and negation were observed in a task of identifying famous faces. In experiments 2 through 4 the question of whether effects of negation were still obtained when effects due to the reversal of pigmentation in negative images were eliminated was examined. By the use of images of the 3-D surfaces of faces measured by laser, and displays as smooth surfaces devoid of pigmentation, only effects of inversion were obtained reliably, suggesting that the effects observed in experiment 1 arose largely through the inversion of pigmentation values in normal images of faces. The results of experiment 5 suggested that the difference was not due to the different task demands of experiments 2-4 compared with those of experiment 1 . When normally pigmented face images were used in a task making similar demands to that of experiment 4 , independent effects of inversion and negation were again observed. When a task of sex classification was used in experiments 6 and 7 , clear effects of negation as well as inversion were observed on latencies, though not accuracies, of responding. The results are interpreted in terms of the information content of pigmentation relative to shape from shading in different face-classification tasks. The results also reinforce other recent evidence demonstrating the importance of image intensity as well as spatial layout of face 'features'.
\end{abstract}

\section{Introduction}

Recent research into the processes involved in face recognition has resulted in good progress in our understanding of the cognitive processes involved, but rather less success in understanding the perceptual representation of faces that allows individual faces to be identified (eg see Bruce and Young 1986; Bruce 1988; Young and Bruce 1991 for reviews). Studies of the effects of inversion on face perception and recognition suggest that face processing involves the encoding of the relationships between one facial 'feature' and another, as a configuration ${ }^{(1)}$, in addition to any coding of features in isolation. In this paper we examine the effects of inversion alongside a much less studied transformation, that of 'negation' of the facial image. We use the results of our experiments to argue that representations mediating face classification rely upon pigmentation and shading patterns in addition to the spatial layout of face features.

There is considerable converging evidence both that isolated features, and that the overall configuration of features, are important in the representation of faces (eg Sergent 1984; Rhodes et al 1993; Tanaka and Farah 1993). Configural processing seems to be particularly disrupted by inversion of faces. A dramatic demonstration of this was given by Young et al (1987), who showed how the alignment of top and bottom halves of different famous faces produced a composite from which it was extremely difficult to name the individual components, compared with a condition

(1) In this paper we will not address the tricky question of the exact distinction between face 'feature' and face 'configuration'. It is enough for present purposes to note that the relationship between different local properties of faces ('features') seems to be important in face identification and to be disrupted by inversion. 
where the two halves were misaligned. However, when the composite images were inverted, performance on naming each half actually improved. They argued that configural processing of the new configuration, which was provided by the composite, was impaired when the display was inverted, allowing the components of each half to be more accurately identified. Similarly, in the 'Thatcher illusion' (Thompson 1980), the eyes and mouth of a face are inverted within the face, to provide an image which looks grotesque when viewed upright, but which looks normal when viewed upside down. Bartlett and Searcy (1993) provided evidence that the illusion is explained by the failure to process the configural relationships between the features when the whole image is presented upside down.

The disproportionate effect of inversion on the recognition of faces compared with other materials (Yin 1969; Scapinello and Yarmey 1970) may arise because configural processing of the precise relationships between different components is particularly important for discriminating between members of categories which share the same overall configuration. Diamond and Carey (1986) showed that people who were expert at a different task of discriminating exemplars from a structurally homogeneous category-dog judges-were also disproportionately impaired at identification of the dogs when these were shown upside down.

Experiments on the effects of inverting facial images have clearly been important in helping us to understand the nature of the representational processes which underlie face identification. Another manipulation which appears to disrupt face recognition badly, but which has been much less adequately researched (Goldstein and Chance 1981 ) is 'negation', where images are displayed as photographic negatives with the original brightness levels reversed (eg Galper 1970; Galper and Hochberg 1971; Phillips 1972). The disruptive effect of negation is intriguing, since a photographic negative preserves the spatial layout and configuration of all the face 'features' that might form candidate representational primitives (eg distance between eyes, ratio of nose length to face length, and so forth). Hayes (1988) and Hayes et al (1986) have demonstrated that negation only disrupts recognition of images containing relatively low spatial frequency information. High-pass filtered images of faces, or line drawings without any shaded or pigmented blocks, are recognised as readily from negative as from positive versions. However, line-drawn faces are themselves poor representations for recognition (Davies et al 1978; Bruce et al 1992) a point we return to in section 10. These observations suggest that photographic negatives are difficult because face recognition depends in part on information conveyed in slow-varying intensity patterns, over and above that conveyed by the spatial layout of major contours which is preserved in high spatial frequency or line-drawn images.

There are two plausible candidates for what the information might be that is conveyed by slow-changing intensity patterns and is affected by negation. The first possible information source is what we will term 'pigmentation'(2), by which we mean skin and hair colour and variations in these. A negative image depicts dark hair as white and white hair as dark, for example. A second possible information source is the pattern of shading and shadow which may help specify the three-dimensional (3-D) shape of the face (cf Bruce 1988). Johnston et al (1992) provided some evidence that shape from shading may contribute to identification by showing that the effect of inversion is reduced if faces are lit from below. An inverted bottom-lit face presents a top-lit image, and Johnston et al suggest that this makes it easier to derive

(2) This is not an ideal term to capture variations in skin and hair colour and texture, but we prefer it to the alternatives: 'colouration' seems confusing when in all our work we have used monochrome images, and 'texture' (which would be the appropriate label within the imageprocessing community) seems to us confusing as its everyday meaning would suggest roughness or coarseness of skin or hair, rather than relative lightness and darkness. 
3-D shape information which can partially compensate for the impairment of configural processing. An image of a face in photographic negative cannot be interpreted correctly in terms of shape from shading. Although a photographic negative of a normal (ie top-lit or front-lit) face resembles an image obtained from a different direction of lighting, it is subtly different. Ignoring the reversal of pigmentation, a negative of a top-lit face resembles a bottom-lit face because lower areas which were dark in the positive version will be bright in the negative version. However, if a light source is lowered this has little effect on the relative brightness around the edge of the face but these areas are also reversed in a photographic negative. Thus if patterns of shading are used to recover 3-D shape, a negative image of a particular face cannot be interpreted in terms of its correct geometry.

If either or both of these potentially distinct information sources (pigmentation and shading) is important for face identification, over and above the spatial layout of facial 'parts', we should expect the effects of negation to be independent of those of inversion when these two manipulations are combined. Bruce et al (1993) used this reasoning when examining the separate and combined effects of inversion and negation on accuracy of decisions about the sex of faces. Other research reported by Bruce et al (1993; also Roberts and Bruce 1988) suggested that sex judgments involved both configural and 3-D shape processing. We therefore suggested that if inversion impaired configural processing, and negation affected processing of 3-D structure via shape from shading, the combined effects of the two manipulations should be greater than the effects of either manipulation alone. This result was found in a task where subjects had to classify the sex of faces shown with eyes closed and with hair concealed by a swimming cap. These conditions of presentation made the task difficult enough to avoid ceiling performance, which could result if hairstyle and hair length were available as cues for this task, but also had the effect of minimising pigmentation cues which might have contributed to the effects of negation. Moreover, whereas pigmentation may be relevant for identity, it is not relevant for classifying sex, since hair colour does not help in telling male from female faces, so it is hard to see why the effect of negation seen in this task should be due to reversal of pigmentation.

Kemp et al (1990) also examined the independent and combined effects of inversion and negation on a perceptual task in which subjects had to decide whether or not face features had been displaced. They found that subjects were impaired both by negation and by inversion, and that the combined effects of the two manipulations exceeded the effect of either alone. They concluded that this implied that there were different sources for the two effects of inversion and negation, and in further experiments were able to dissociate further effects of negation from those of inversion. In particular, they found that when internal face features were replaced with dots, effects of negation were still observed on discrimination accuracy but effects of inversion were not.

The results of these experiments thus suggest that inversion and negation affect different aspects of face perceptual processing. However, in neither of the earlier studies were the effects of these two manipulations on a task involving facial identification assessed. The face is used to derive a number of different kinds of meaning, and there is good evidence that some face-processing routes proceed independently of others (Bruce and Young 1986; Young and Bruce 1991). In order to learn more about the different sources of information used to specify identity, it is important to use tasks which tap the identification route directly. Identification tasks have been used in few previous investigations of effects of negation. For example in their early research Galper and colleagues used recognition memory for previously unfamiliar faces, which confounds picture memory with face recognition (Bruce and Young 1986). 
In our first experiment we explored whether negation affected identification of familiar faces independently of inversion. This manipulation was contained within the second experiment reported by Johnston et al (1992), but their experiment was complicated by the further manipulation of direction of lighting, and the complex interaction between all three factors obscured the relationship between effects of inversion and negation alone. In experiment 1 we investigated this using a simpler design. We then went on to investigate whether the effect of negation on identification tasks is due in any part to the reversal of shading patterns. This was achieved by examining whether an effect of negation can still be observed when any effects due to reversal of pigmentation have been eliminated. These studies were made possible by a novel method for presenting the 3-D surfaces of faces measured by laser.

\section{Experiment 1}

The aim in this experiment was to see whether negation affected identification of faces independently of inversion, which would be consistent with these two transformations disrupting different aspects of face processing. However, in order to explore the individual and combined effects, it is necessary to find a task which does not suffer from floor effects (if the effect of inversion takes performance to chance, we cannot look for any further decrement due to negation) or from ceiling effects (where neither inversion or negation alone produce decrements). In pilot work we found that free identification of faces produced floor effects, but we were able to develop a satisfactory task which avoided this problem, where subjects had to identify, within a fixed time limit, famous faces from a list of named alternatives (see also Valentine and Bruce 1988; Bruce et al 1991).

\subsection{Method}

2.1.1 Subjects. These were sixteen undergraduate and postgraduate students. Each received $£ 1$ for participating in the experiments, which took about $15 \mathrm{~min}$.

2.1.2 Materials and apparatus. Twenty-eight black-and-white photographs of people (majority male) likely to be familiar to these subjects were selected from a larger pool of items. The faces were all shown in full-face or three-quarter view and copied onto slides in which each head appeared in a circular window which concealed all background information. Each slide was prepared both in photographic positive and in photographic negative versions. Inversion was achieved in this and all other experiments by rotating the faces $180^{\circ}$ within the plane. The slides were presented on a Bell and Howell back-projection apparatus with a $5 \mathrm{~s}$ presentation time for each slide.

2.1.3 Design. This was a within-subjects design with 4 conditions formed by 2 orientations (upright and inverted faces) $\times 2$ polarities (positive and negative images). Each subject saw 7 faces in each of the four conditions of the design. Each face was presented in each of the 4 experimental conditions 4 times, as sets of 7 faces were rotated around experimental conditions between subgroups of four subjects.

2.1.4 Procedure. The subjects were given approximately 2 min to study a list of the names of the celebrities whose faces, they were told, would appear in the experiment. The subjects were then asked simply to name the face that appeared on the screen, and they were warned that some of the faces would be presented in photographic negative, some inverted, and some negative and inverted. Each slide was presented for $5 \mathrm{~s}$ and subjects were asked to make their response within this time limit. The subjects' responses were noted and scored for accuracy. Failure to respond within the $5 \mathrm{~s}$ resulted in an 'incorrect' score for that slide. Items were presented in a random order to each subject. 


\subsection{Results}

In table 1 the mean number of items correctly identified within $5 \mathrm{~s}$ in each condition of the experiment is shown. Inversion and negation both reduce accuracy compared with the untransformed condition, and the two transformations combined had a greater effect than had either transformation alone. These observations were supported by a 2 (orientation) $\times 2$ (polarity) ANOVA. This revealed a main effect of orientation $\left(F_{1,15}=44.61, p<0.01\right)$, and a main effect of polarity $\left(F_{1,15}=58.76\right.$, $p<0.01)$. The interaction term did not reach significance $\left(F_{1,15}<1\right)$. Thus the results show additive effects of negation and inversion.

Table 1. Percent correct identification of famous faces in each condition of experiment 1.

\begin{tabular}{lll}
\hline Orientation & Image & \\
\cline { 2 - 3 } & positive & negative \\
\hline Upright & 94.6 & 55.4 \\
Inverted & 70.4 & 25.1 \\
\hline
\end{tabular}

\subsection{Discussion}

The significant and additive effects of negation and inversion found in this experiment are consistent with the suggestion that negation specifically affects a different set of processes from those affected by inversion. ${ }^{(3)}$ The size of the negation effect observed here is notable. Even though negative images preserve the spatial layout of all the facial 'features', negative images were identified at rates some $40 \%$ below those achieved with positive images. Indeed, the effects of negation in this experiment are greater than are those of inversion. The results appear generally to be consistent with those obtained by Johnston et al (1992) with top-lit faces, where inversion and negation both appeared to reduce performance on a face-familiarity decision task (expressed as an index of sensitivity) to about the same extent and independently of each other.

These results are also consistent with those of Kemp et al (1990) who observed independent effects of negation and inversion in a study of sensitivity to feature displacement in a face-matching task, and with those of Bruce et al (1993) who found additive effects in a sex-classification task. However, as we noted in section 1 , the effects of negation may arise because of the reversal of pigmentation levels, or patterns of shading, or both. The reversal of pigmentation levels should be irrelevant in the Kemp et al (1990) studies, since the task required only the detection of a displacement in the facial features. Moreover, it would be difficult to understand why altering pigmentation levels should make it harder to classify the sex of faces. Johnston et al (1992) discussed the contribution of pigmentation effects to the negation effect, but did not explore it further. In the remaining experiments described in this paper, we make use of novel methods for displaying faces devoid of pigmentation in order to assess the role played by shading alone in the negation effect, separately for tasks requiring identification (experiments 2-4) and sex classification (experiments 6 and 7).

(3) Bundesen (personal communication) has pointed out that it is difficult to argue for the independence of underlying processes from additivity of accuracy scores as opposed to latencies. This is because if each independent process has a certain probability of success (or failure) the combined probability of success (or failure) should be the product of multiplying, rather than adding, the independent probabilities. However, in these tasks we have not got 'pure' accuracy data but instead have measured performance within a time deadline of $5 \mathrm{~s}$, which means our measure is a hybrid speed/accuracy measure. 


\section{Materials used in experiments $2-4,6$, and 7}

The materials used in experiments $2,3,4,6$, and 7 were obtained as a result of a collaboration with a team, working on surgical reconstruction of faces, at University College Hospital [eg see Linney (1992) for a description of the project and scanner]. The elimination of pigmentation cues was achieved by making use of pictures of facial structures produced by measurement of the 3-D surface with a laser. The technique is described in detail elsewhere (eg Bruce et al 1991, 1993). Briefly, the subject is rotated in front of a laser beam which projects a line onto the face to measure the contour of the face profile at that point, and this measurement is repeated for a number of different profiles, spaced more closely around the face than around the back of the head. For the experiments reported here, hair was concealed with a stocking during the scan since hair reflects the laser very erratically. The results of such scanning provide some $20000 x, y, z$ coordinates which describe the facial surface in great detail. These coordinates (or a subset of them) can be used to construct a very high-density polygonal mesh, which can be displayed as a smooth surface by presenting these facets to a notional light source with the use of standard 3-D computer graphics techniques. Facial surfaces revealed in this way, after scanning at University College London and display of the resulting images are shown in figure 1. Images of faces created in this way were trimmed to remove the topmost portion of the head from which laser reflection was erratic. During the course of our collaborative project a number of people, whose faces would be familiar to subjects in Nottingham, visited University College London to have their faces laser scanned, which allowed us to design the identification experiments reported here and in Bruce et al (1991).

For the experiments reported here we used a Phong diffuse-shading algorithm which models the effect of a single distant light source on a matte surface, with no specular component. The position of the light source used in all the experiments with surface images remained constant, from a direction above and slightly to the left of the viewer's line of sight (ie as though it came from above the viewer's left shoulder). This direction meant that images of faces facing in a left direction received illumination from a light source shining fairly directly onto the face, full-face images received illumination from an angle, and heads facing in a right direction had their faces partly in shadow (see figure 1). This lighting position meant that the images of left-facing and right-facing heads were less symmetrical, increasing the differences between different exemplars of the same person's head. ${ }^{(4)}$

\section{Experiment 2}

In this experiment we investigated the independent and combined effects of negation and inversion on a task of discriminating one individual's head from another, when these were portrayed as surfaces of the type shown in figure 1 . In an attempt to minimise the use of 'pictorial' cues to task solution (cf Bruce and Young 1986), the viewpoint in which heads were shown was varied from left through to right threequarter views with a lighting direction that maximised differences between left-facing and right-facing heads.

(4) In the analyses presented of experiments 2, 3, 4, 6, and 7 we have not investigated the effect of the angle of the head as a separate factor. However, we have checked in additional analyses of experiments 3 and 4 that there is no differential effect of negating left-facing versus right-facing heads. These analyses reveal no statistically significant differences between the recognition of the left-facing and right-facing heads (though a nonsignificant trend in each experiment for those with more-direct illumination to be better recognised), and no interaction or trend towards an interaction between direction of head and whether positive or negative images were shown (all $p$ s $>0.3$ ). 

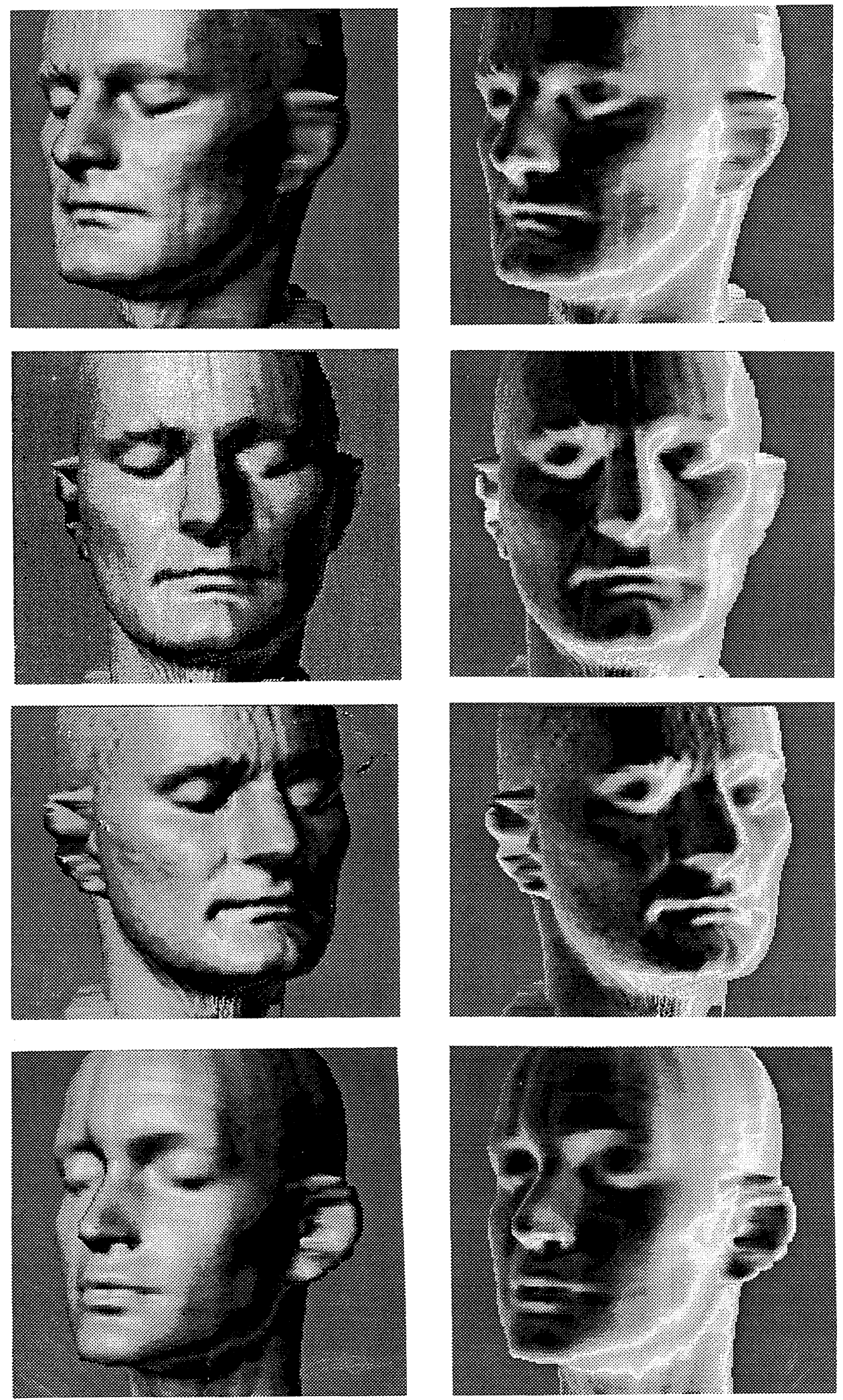

Figure 1. Examples of the surface images of faces used in the reported experiments. The figure shows positive (left) and negative (right) examples of $-45^{\circ}$ (top row), full-face (second row) and $+20^{\circ}$ images (third row) of one of the male faces used in experiments $2-4$, and the $-45^{\circ}$ view of one of the female faces used in experiments 6 and 7 (bottom row). 
4.1 Method

4.1.1 Subjects. Twenty subjects took part in the experiment. All subjects were tested on the stimuli portraying faces of JB and PL, but as three of the subjects were not familiar with the faces of NS and MB only seventeen were tested on images of these.

4.1.2 Materials. Laser scans of four individuals' heads were used as target faces in the experiment. All were male and current or recent members of staff in the Psychology department at Nottingham University and are labelled as JB, PL, NS, and MB. Images of these heads were prepared with the use of a SUN 3/110 greyscale workstation and displayed at 5 different head angles $\left(-45^{\circ},-20^{\circ}, 0^{\circ},+20^{\circ}\right.$, and $+45^{\circ}$ angles where $0^{\circ}$ is full face and $45^{\circ}$ is a three-quarter view). The resulting images were then transferred to a Macintosh IIcx with a 256 grey-level monitor, where upright/positive, upright/negative, inverted/positive, and inverted/negative images of each head were created at each orientation with the aid of image-processing software.

The stimuli were presented to subjects by means of a tachistoscope software package written for the Mac IIcx.

4.1.3 Design and procedure. This was a within-subjects design with 4 conditions formed by all combinations of 2 orientations (upright and inverted) $\times 2$ polarities (positive and negative).

The experiment was divided into two blocks, which formed partial replications of the experiment with different pairs of heads. In one block the subjects were shown all the manipulations of heads JB and PL and asked to respond to the identity of the stimulus by depressing one key for JB and another for PL. In the other block, heads NS and MB were presented and the task was to discriminate between these. Thus in each block, subjects were presented with 2 heads $\times 2$ orientations (upright and inverted) $\times 2$ polarities (positive and negative) $\times 5$ views $\left(-45^{\circ},-20^{\circ}, 0^{\circ},+20^{\circ}\right.$, $+45^{\circ}$ ), to give a total of 40 stimulus presentations.

The heads were presented in a random order with the aid of the tachistoscope program, with each head displayed until the subject had made a response. There was then a $1 \mathrm{~s}$ blank field and then the next stimulus face. Reaction times were recorded in addition to response accuracy. Responses and latencies were recorded from the depression of one of two keys on the Macintosh keyboard which were assigned to the two response alternatives. Subjects were instructed to press the appropriate key on each trial as quickly as possible, without sacrificing accuracy. A short series of 10 practice trials was given to each subject before each block. In these trials the name of each stimulus head was used instead of the 3-D laser head, and these were presented for the subjects to gain familiarity with the response mappings.

Volunteers were recruited into this experiment only if they claimed familiarity with the faces of JB and PL, and preferably of NS and MB in addition. It proved impossible to recruit twenty subjects who claimed confidence with all four faces. All twenty subjects were familiar both with PL and JB but three subjects were unfamiliar with either MB or NS and as a consequence the MB/NS task was presented to only seventeen subjects.

\subsection{Results}

In table 2 the accuracy and latency scores averaged across subjects (twenty for the $\mathrm{JB} / \mathrm{PL}$ discrimination; seventeen for the MB/NS discrimination) are shown. There is a clear effect of orientation, with performance in both tasks less accurate, and slower, for inverted than for upright faces. However there appears to be little effect of negation on either dependent variable, in either block of trials. These observations were confirmed in separate 2 (orientation) $\times 2$ (polarity) ANOVAs which were conducted on the data from each block, both for accuracy and for latencies. 
For the PL/JB block the analyses revealed main effects of orientation both for accuracy and for latency data $\left(F_{1,19}=78.88, p<0.01\right.$ and $F_{1,19}=10.95, p<0.05$, respectively). Neither the main effects of polarity nor the interaction between orientation and polarity reached significance for either reaction-time or accuracy data. However, in the analysis of accuracies the effect of polarity approached significance $\left(F_{1,19}=3.02, p=0.1\right)$ (all remaining $F$ s in these analyses $<=1$ ). The pattern of results was very similar for the NS/MB block with main effects of orientation both for accuracy and for reaction times $\left(F_{1,16}=33.48, p<0.01\right.$ and $F_{1,16}=16.43, p<0.01$, respectively). The effect of polarity and the interaction terms failed to approach significance either for accuracy or for latency data (all $F_{\mathrm{S}}<1$ ).

Table 2. Mean percent correct responses, and correct-decision latencies (in milliseconds) (shown in parentheses) in experiment 2.

\begin{tabular}{llll}
\hline $\begin{array}{l}\text { Discrimination } \\
\text { task }\end{array}$ & Orientation & \multicolumn{2}{l}{ Image } \\
\cline { 3 - 4 } & & positive & negative \\
\hline JB/PL & Upright & $93.0(915)$ & $86.0(966)$ \\
& Inverted & $72.5(1256)$ & $69.0(1299)$ \\
MB/NS & Upright & $86.5(1027)$ & $86.5(1029)$ \\
& Inverted & $67.1(1229)$ & $64.7(1242)$ \\
\hline
\end{tabular}

\subsection{Discussion}

Surprisingly, no significant effects of negation were found in any part of this experiment, whereas inversion had a strong detrimental effect both on accuracy and on latency of discrimination for both pairs of heads. Note that the experiment was sensitive enough to detect effects of inversion, and so it cannot be the case that the absence of negation effects is due to lack of power in the design. One possible reason for the absence of any effect of negation is that subjects may have used some strategy to make the discrimination between the heads in each pair based on some local feature, such as the size or shape of the chin, even though we varied viewpoint and image properties in an attempt to discourage such strategies. This type of strategy might be disrupted by inversion but not necessarily by negation. In experiment 3 we repeated the study but made the discrimination less easy to perform on the basis of single local features, by requesting that subjects discriminate one pair of heads from the other.

\section{Experiment 3}

This was similar in conception and design to experiment 2 , but subjects were now asked to classify each head as belonging to one or other pair of identities.

\subsection{Method}

5.1.1 Subjects. Fifteen subjects participated in this experiment; all were members of staff or students in the Psychology department and as a result were familiar with the members of staff whose heads appeared as stimuli in the experiment.

5.1.2 Materials, design, and procedure. The same materials as those used in experiment 2 were used in a within-subjects design with 4 conditions formed by all combinations of 2 orientations (upright and inverted) $\times 2$ polarities (positive and negative). Thus each subject saw 4 heads $\times 2$ orientations (upright and inverted) $\times 2$ polarities (positive and negative) $\times 5$ viewpoints, to give a total of 80 stimulus presentations.

The heads were presented in a random order with the aid of a tachistocope program, with each head displayed until the subject had made a response using one of two keys. Subjects were instructed to press one key if the head was that of JB or NS 
and the other if it was MB or PL. There was then a $1 \mathrm{~s}$ blank field and then the next stimulus face. As in experiment 2, a short series of 10 practice trials was given to each subject before the experiment, in which the names of the faces to be used in the experiment proper were used. These trials were to familiarise the subject with the key presses required.

\subsection{Results}

The average accuracy and latency in each condition of the experiment is shown in table 3. Again there are clear effects of inversion, but rather little apparent effect of negation in this task.

Two $2 \times 2$ ANOVAs were conducted on the data, with orientation and polarity as the within-subjects factors, and subjects as the random factor. The ANOVA conducted on the accuracy data revealed a significant effect of orientation $\left(F_{1,14}=38.47\right.$, $p<0.001)$ but the effect of polarity failed to reach significance $\left(F_{1,14}=2.28\right.$, $p=0.15)$. There was no interaction $(F<1)$. The analysis of the reaction-time data revealed a main effect of orientation $\left(F_{1,14}=6.99, p<0.05\right)$ but no main effect of polarity or interaction $\left(F_{\mathrm{s}}<1\right)$.

Table 3. Mean percent correct responses and correct-decision latencies (in milliseconds) (shown in parentheses) in experiment 3.

\begin{tabular}{lll}
\hline Orientation & Image & \\
\cline { 2 - 3 } & positive & negative \\
\hline Upright & $82.3(1194)$ & $76.0(1201)$ \\
Inverted & $63.0(1358)$ & $61.3(1399)$
\end{tabular}

\subsection{Discussion}

The results of this experiment show significant effects of inversion but not of negation. Clearly our design was powerful enough to reveal a highly significant effect of inversion. Although there was a trend for negative images to be less accurately identified than positive images this effect was far from statistically significant.

Our preliminary conclusion, from the results of experiments 2 and 3 together, is that the effect of negation which we obtained in experiment 1 arose largely because of the effects on pigmentation. Once pigmentation cues are eliminated, there is rather little residual effect of negation as shading patterns are not important for face identification, so that their reversal from normal does not impede performance. However, it is possible that the task used in experiment 3 was still not sufficiently demanding of the facial-recognition system, and subjects may have adopted particular strategies to achieve the somewhat arbitrary response mappings. In the next experiment, we moved to a task in which we presented a still-larger set of heads in a task more analogous to that of discriminating known from unknown faces.

\section{Experiment 4}

\subsection{Method}

6.1.1 Subjects. Sixteen subjects participated in this experiment; all were members of staff or students in the Psychology department who were familiar with the members of staff whose heads were used as stimuli in the experiment.

6.1.2 Materials, design, and procedure. The 3-D representations of 8 male individual heads were used as stimuli in the experiment. The same 4 familiar heads were used as in the previous experiment, ie JB, PL, NS, and MB. The other 4 heads used were all male and selected to be unfamiliar to members of the department. 
All the heads were Phong shaded and prepared at $-45^{\circ},-20^{\circ}, 0^{\circ},+20^{\circ}$, and $+45^{\circ}$ viewpoints. The heads were then viewed under 2 orientations (upright and inverted) $\times 2$ polarities (positive and negative) to give the 4 cells of the design. Thus each subject was presented with 8 heads $\times 2$ orientations $\times 2$ polarities $\times 5$ viewpoints, a total of 160 stimulus presentations.

The stimuli were divided by viewpoint into two blocks of 80 heads so that one block included the $-45^{\circ}, 0^{\circ}$, and $+45^{\circ}$ viewpoints of 4 of the heads and the $-20^{\circ}$ and $+20^{\circ}$ viewpoints of the other 4 heads. The second block contained the complementary combination of heads and viewpoints. Within each block each head was presented under all of the orientation/polarity conditions.

The materials were presented with the aid of the same equipment and general procedure as in experiments 2 and 3. However, rather than asking subjects to discriminate between particular familiar heads, subjects were asked to depress one key if the stimulus head was familiar (ie any of JB, PL, NS, or MB) and another key if it was unfamiliar. The subjects were told the names of the familiar heads that could appear in the instructions for the experiment, in order to keep performance above floor levels (cf Bruce et al 1991).

Reaction times and percentage of accurate responses were recorded as dependent variables in the experiment.

\subsection{Results}

The accuracy and latency data of the performance to the 4 familiar faces in this experiment (ie the 'hits') are summarised in table 4a for comparability with the performance in experiments 2 and 3 , which involved responses to only this same set of 4 faces.

Two $2 \times 2$ ANOVAs were conducted on the 'hits' data with orientation and polarity as the within-subjects factors.

The ANOVA conducted on the accuracy data revealed a significant main effect of orientation $\left(F_{1,15}=22.88, p<0.001\right)$ and a significant main effect of polarity $\left(F_{1,15}=20.65, p<0.001\right)$ with no interaction $(F<1)$.

The analysis of the reaction-time data yielded a significant main effect of orientation $\left(F_{1,15}=15.69, p<0.05\right)$ with upright heads receiving faster responses than inverted heads. The effect of polarity failed to reach significance $\left(F_{1,15}=2.64\right.$, $p=0.11)$. There was no interaction $(F<1)$.

Table 4. (a) Mean percent correct identifications and correct-decision latencies (in milliseconds) (shown in parentheses) to familiar faces in experiment 4.

\begin{tabular}{lll}
\hline Orientation & \multicolumn{2}{l}{ Image } \\
\cline { 2 - 3 } & positive & negative \\
\hline Upright & $79.4(962)$ & $69.7(1030)$ \\
Inverted & $55.6(1262)$ & $44.7(1302)$ \\
& \\
\hline \\
(b) Mean percent false-positive identifications of unfamiliar faces, and $d^{\prime}$ scores (shown in \\
parentheses) in experiment 4. \\
\hline
\end{tabular}

\begin{tabular}{lll}
\hline Orientation & Image & \\
\cline { 2 - 3 } & positive & negative \\
\hline Upright & $15.3(2.1)$ & $10.3(2.1)$ \\
Inverted & $32.2(0.7)$ & $21.3(0.8)$ \\
\hline
\end{tabular}


Analysis of the accuracy data from familiar faces alone, therefore, appears to reveal a significant effect of negation, independent of that of inversion. However, this pattern is not maintained once data from the unfamiliar faces are considered. In table $4 \mathrm{~b}$ the false-positive data for the 4 unfamiliar faces are shown and these clearly indicate that in the negated conditions, where familiar faces are poorly recognised, unfamiliar faces are accurately detected and yield a low false-positive rate. If hit rates are combined with false-positive rates to give $d^{\prime}$ scores (table 4b), an ANOVA on these reveals a main effect of orientation $\left(F_{1,15}=37.5, p<0.001\right)$ but no effect of negation nor any interaction $\left(F_{\mathrm{S}}<1\right)$. It therefore appears as though the apparent effect of negation on the familiar faces may have arisen because of a response bias which has led subjects to respond "unfamiliar" to negative images, rather than because of a problem of discrimination of identity per se.

\subsection{Discussion}

In contrast to the results of experiments 2 and 3 , here there do appear to be significant effects of negation, but they are rather curious. Negative images of the familiar heads are harder to identify as familiar, but negative images of the unfamiliar heads are easier to classify as unfamiliar. It therefore seems that negation is having the effect of biasing responses towards the unfamiliar. The results of experiments 2 to 4 , taken together, suggest that although we cannot say there are no effects of negating surface images on their identification (there were small trends in the predicted direction in all our studies), it seems clear that the enormous effects of negation found with famous faces in experiment 1 are largely absent once heads devoid of pigmentation are used. The results of these experiments seem to provide the example requested by Kemp et al (1990) of a manipulation which will reveal an inversion effect without an effect of negation.

However, it is possible that the differences are not due to the absence of pigmentation in the laser heads, but to the relatively small numbers of items used in the experiments. To check whether the large and reliable effects of negation we found in experiment 1 could be found with smaller item sets, like that of experiment 4 , we repeated experiment 4 with normal photographs of faces.

\section{Experiment 5}

\subsection{Method}

7.1.1 Subjects. Twelve subjects participated in this experiment; all were members of staff or students in the Psychology department at Nottingham and were familiar with the faces of four ex-members of the same department who had moved to the University of Stirling.

7.1.2 Materials, design, and procedure. A total of 8 individual faces were used as stimuli in the experiment. There were 4 faces that would be familiar in Nottingham ( 3 male, 1 female) and 4 were of people unfamiliar in Nottingham ( 3 male, 1 female). All male faces were clean shaven, and the unfamiliar people were chosen to be fairly similar in age and with similar kinds of hairstyle to the individuals who were familiar to the subjects (for example, the familiar and the unfamiliar female were both $30-40$ years old, and had dark curly hair). Pictures of all 8 people were obtained by grabbing individual frames from a video camera showing each person in $-45^{\circ},-20^{\circ}, 0^{\circ}$, $+20^{\circ}$, and $+45^{\circ}$ viewpoints. Lighting was diffuse natural light from a window slightly above the level of the heads as they were digitised. The 5 pictures of each person were then prepared under 2 orientations (upright and inverted) $\times 2$ polarities (positive and negative) to give the 4 cells of the design. Thus each subject was presented with 8 heads $\times 2$ orientations $\times 2$ polarities $\times 5$ viewpoints, a total of 160 stimulus presentations. 
The stimuli were divided by viewpoints into two blocks of 80 heads so that one block included the $-45^{\circ}, 0^{\circ}$, and $+45^{\circ}$ viewpoints of 4 of the heads and the $-20^{\circ}$ and $+20^{\circ}$ viewpoints of the other 4 heads. The second block contained the complementary combination of heads and viewpoints. Within each block each head was presented under all of the orientation/polarity conditions.

As in experiment 4, subjects were asked to depress one key if the stimulus face was familiar and another key if it was unfamiliar. The subjects were told the names of the familiar heads that could appear in the experiment.

Reaction times and percentage of accurate responses were both recorded as dependent variables in the experiment.

\subsection{Results and discussion}

The results of the experiments are summarised in table 5, for direct comparability with table 4. The first thing to note is that, whereas the effect of inversion and negation on recognition of familiar faces look very similar to those found in experiment 4 , we now find that the false-positive rates are high when hit rates are low, and the effects of negation appear on $d^{\prime}$.

Analysis of the accuracy scores to familiar faces alone reveals main effects of orientation $\left(F_{1,11}=9.87, p<0.01\right)$, polarity $\left(F_{1,11}=32.9, p<0.001\right)$, and a significant interaction between these $\left(F_{1,11}=5.6, p<0.05\right)$ which seems to arise because of a marginal ceiling effect with positive images in this experiment reducing the normal effects of inversion. Analysis of latencies, time taken to recognise the familiar faces correctly, gives two main effects and no interaction (orientation: $F_{1,11}=23.9$, $p<0.001$; polarity $F_{1,11}=99.4, p<0.001$ ), interaction, $F_{1,11}=1.1$ ). Analysing $d^{\prime}$ yields a similar pattern. There are significant effects of orientation $\left(F_{1,11}=9.9\right.$, $p<0.01)$, polarity $\left(F_{1,11}=24.4, \mathrm{p}<0.001\right)$, and no interaction between these factors $\left(F_{1,11}=1.8, p=0.21\right)$.

It therefore appears that the failure to find effects of negation on $d^{\prime}$ when testing the surface images in experiments $2-4$, was not simply due to the different task demands of discriminating a small set of familiar terms. Our use of photographs of faces in the same task as experiment 4 has given rise to a pattern of data just like that found in experiment 1 . The percentage decrements produced by inversion and negation cannot be compared directly with those in experiment 1 , because of their very different chance levels (chance here was $50 \%$ correct but in experiment 1 it was only $3.6 \%$ correct).

Table 5. (a) Mean percent correct identifications and correct-decision latencies (in milliseconds) (shown in parentheses) to familiar faces in experiment 5.

\begin{tabular}{lll}
\hline Orientation & Image & \\
\cline { 2 - 3 } & positive & negative \\
\hline Upright & $92.1(580)$ & $84.2(701)$ \\
Inverted & $90.0(679)$ & $69.6(838)$ \\
\hline
\end{tabular}

(b) Mean percent false-positive identifications of unfamiliar faces, and $d^{\prime}$ scores (shown in parentheses) in experiment 5 .

\begin{tabular}{lcr}
\hline Orientation & \multicolumn{1}{l}{ Image } \\
\cline { 2 - 3 } & positive & negative \\
\hline Upright & $6.7(3.1)$ & $8.3(2.6)$ \\
Inverted & $14.1(2.6)$ & $16.7(1.6)$ \\
\hline
\end{tabular}


The results of experiment 5 compared with those of experiments $2-4$ reinforce our earlier conclusion that pigmentation is more important than is shading for face identification. As we noted in our introduction, the colour of hair, eyes, and brows is likely to be informative in tasks of face identification, and this would explain why it is reversal of pigmentation that seems to explain effects of negation in this case. However, this should not be true for a task of sex classification, where the colour of hair or eyes provides no useful information for the task. Moreover, our previous research has suggested that useful information about sex of faces is conveyed by the 3-D shape of the facial surface, and this information might be gained by the visual system, at least in part, from shading patterns. It is therefore of interest now to use the surface images, in positive and negative versions, in a task of sex classification. Our prediction was that effects of negating the images of the laser heads should emerge in such a task.

\section{Experiment 6}

This experiment follows on from that of Bruce et al (1993) who showed additive effects of negation and inversion when subjects were asked to judge the sex of faces photographed with hair concealed. These results had indicated that our ability to make this classification may be multiply determined by a combination of local textural cues (eg stubbly cheeks etc), relational information (eg the size of nose in relation to the width of the chin), and three-dimensional information (eg the protruberance of the brow and chin or the prominance of the chin/jaw region).

Following Bruce et al (1993) we would expect to find independent effects of negation and inversion in experiment 6 with the use of the laser images of heads, with negation disrupting 3-D shape-from-shading processing and inversion affecting the processing of relational aspects of the face, since both processes are thought to be of importance in the classification of the sex of a face.

\subsection{Method}

8.1.1 Subjects. Twenty undergraduate subjects, who responded to an advertisement, participated in the experiment.

8.1.2 Materials. Laser scans of 8 individuals' heads were used as stimuli in the experiment, 4 males and 4 females, displayed as smooth surfaces with Phong shading at $+45^{\circ}$ and $-45^{\circ}$ orientations. It was three-quarter views that Bruce et al (1993) found yielded the most accurate performance for sex judgments to laser heads.

8.1.3 Design and procedure. This was a within-subjects design with orientation (upright and inverted), polarity (positive and negative), and sex of the 3-D head as the 3 factors. Each subject saw each of the 8 heads at $+45^{\circ}$ and $-45^{\circ}$ viewpoints, in each of the 4 conditions, making a total of 64 stimulus presentations.

The heads were presented in a random order by the use of the tachistoscope software, with each head displayed until the subject had made a response. There was then a $1000 \mathrm{~ms}$ blank field and then the next stimulus face. Reaction times and percentage of accurate sex judgments were both recorded as dependent variables in the experiment.

Subjects were told they would be shown a number of male and female heads from various viewpoints and that some would be inverted and some shown in negative. The subjects were asked to respond as quickly and accurately as possible as to the sex of the image by depressing one of two keys.

\subsection{Results}

The mean percent correct responses, and associated latencies are shown in table 6a. This table also gives the mean latencies for the fifteen subjects remaining after those who failed to score above chance in some cells of the design were excluded. 
A 2 (orientation) $\times 2$ (polarity) $\times 2(\operatorname{sex})$ ANOVA conducted on the accuracy data for all twenty subjects revealed a main effect of orientation $\left(F_{1,19}=25.8, p<0.001\right)$ as well as interactions between sex of head and orientation $\left(F_{1,19}=22.5, p<0.001\right)$ and between sex of head and polarity $\left(F_{1,19}=6.7, p<0.05\right)$. However, there was also a significant 3 -way interaction between sex, orientation, and polarity $\left(F_{1,19}=5.2\right.$, $p<0.05)$. Investigation of this interaction revealed that neither negation nor inversion were having any effects on the accuracy of judging the sex of the female heads but that inversion severely decreased the accuracy of judging the sex both of positive and of negative male heads. Furthermore, negation only caused a decrement to the accuracy in judging male inverted heads. It seems that inverting a male head makes it look more female and negating this image makes it appear even more so. Bruce et al (1993) observed accuracies of around $85 \%$ correct for judging the sex of surface images of heads like those used here. The pattern of accuracies observed in this experiment suggests that there may be some bias towards labelling the upright laser heads "male" and that this bias is being altered by inversion and negation.

In order to get a clearer picture of how accuracy in this task was affected by orientation and negation, the data from male and female faces were combined to give a measure of $d^{\prime}$, where a 'hit' was considered to be a correct judgment of 'male' to a male face, and a 'false positive' an incorrect judgment of 'male' to a female face. The mean $d$ 's computed in this way are shown in table 6 b. A 2 (orientation) $\times 2$ (polarity) ANOVA yielded a significant effect of orientation $\left(F_{1,19}=19.4, p<0.001\right)$, no effect of polarity $(F<1)$, and no significant interaction between these $\left(F_{1,19}=1.99\right.$, $p=0.175)$. This analysis therefore confirmed that there was no significant effect of negation on accuracy in this task.

However, as the overall accuracy averaged across male and female heads approached effective ceiling in some cells of this design it becomes important to analyse the reaction-time data for those responses made accurately.

A 2 (orientation) $\times 2$ (polarity) $\times 2($ sex $)$ within-subjects ANOVA conducted on the reaction-time data for all twenty subjects revealed a main effect of orientation

Table 6. (a) Mean percent correct responses and correct-decision latencies (in milliseconds) (shown in parentheses) in experiment 6. Figures marked with an asterisk indicate mean correctdecision latencies after the exclusion of data from the five least-accurate subjects.

\begin{tabular}{lll}
\hline Orientation & \multicolumn{2}{l}{ Image } \\
\cline { 2 - 3 } & positive & negative \\
\hline Male faces & $96.4(832)\left(868^{*}\right)$ & $96.4(906)\left(961^{*}\right)$ \\
Upright & $75.8(1156)\left(1199^{*}\right)$ & $61.4(1322)\left(1418^{*}\right)$ \\
Inverted & $77.7(972)\left(988^{*}\right)$ & $79.6(997)\left(1040^{*}\right)$ \\
Female faces & $80.2(1095)\left(1140^{*}\right)$ & $87.7(1161)\left(1247^{*}\right)$ \\
Upright & \\
Inverted & 80 \\
\hline
\end{tabular}

(b) Mean $d^{\prime}$ scores obtained by combining correct "male" responses with incorrect "male" responses.

\begin{tabular}{lll}
\hline Orientation & Image & \\
\cline { 2 - 3 } & positive & negative \\
\hline Upright & 3.1 & 3.2 \\
Inverted & 2.2 & 1.9 \\
\hline
\end{tabular}


$\left(F_{1,19}=29.9, p<0.001\right)$ qualified by a significant interaction between sex and orientation $\left(F_{1,19}=18.6, \mathrm{p}<0.001\right)$. However, the main effect of polarity just failed to reach significance on a two-tailed test $\left(F_{1,19}=4.0, p=0.061\right) .{ }^{(5)}$ A second ANOVA was based on the reaction times excluding data from subjects who scored at chance in one or more cells of the design. An ANOVA conducted on the data for the most accurate fifteen subjects revealed a main effect of orientation $\left(F_{1,14}=22.7\right.$, $p<0.001$ ) showing that inverted heads are classified much more slowly than are upright heads. The main effect of orientation was qualified by a significant interaction between sex of the 3-D head and its orientation $\left(F_{1,14}=13.7, p<0.01\right)$. A main effect of polarity $\left(F_{1,14}=5.3, p<0.05\right)$ shows that negating a head also slows down response times. No other interaction terms reached significance (all $p \mathrm{~s}>0.1$ ), indicating that the effects of negation and inversion are in this case additive.

\subsection{Discussion}

The results of this experiment reveal effects of negation as well as inversion, at least for the reaction-time data, though the effect of negation was clearer when the leastaccurate subjects were excluded. Moreover, the results of the analysis of accuracy data were rather complex. We decided to replicate this experiment, with a rather larger sample of heads, in order to discover whether the trends found in experiment 6 were robust.

\section{Experiment 7}

\subsection{Method}

9.1.1 Subjects. Sixteen subjects took part; all were postgraduates and employees at the university responding to an advertisement for research volunteers.

9.1.2 Materials, design, and procedure. This was the same as experiment 6 , except that laser scans of 16 individuals' heads were used as stimuli-8 males and 8 females. Each was prepared in both $+45^{\circ}$ and $-45^{\circ}$ views, and shown in each of the 4 conditions of orientation and polarity to yield a total of 128 trials in this experiment.

\subsection{Results}

The mean percent correct responses and corresponding mean reaction times are shown in table 7a. A 2 (orientation) $\times 2$ (polarity) $\times 2(\operatorname{sex})$ ANOVA on the accuracy data revealed a main effect of orientation $\left(F_{1,15}=48.6, p<0.001\right)$ and a significant interaction between sex of head and orientation $\left(F_{1,15}=12.66, p<0.005\right)$. Further analysis of this interaction indicated that inversion significantly reduced the accuracy of judging the sex of male, but not of female, heads. No other effects reached significance (for effect of polarity, $F_{1,15}=2.08, \mathrm{p}>0.1$ ). As in experiment 6 , the correct and incorrect "male" responses were combined to give measures of $d^{\prime}$ and the average $d^{\prime}$ scores are shown in table $7 \mathrm{~b}$. A 2 (orientation) $\times 2$ (polarity) ANOVA gave a significant main effect of orientation $\left(F_{1,15}=57.5, p<0.001\right)$ and a marginal effect of polarity $\left(F_{1,15}=4.35, p=0.051\right.$, two-tailed $)$ and interaction between orientation and polarity $\left(F_{1,15}=3.42, p=0.08\right)$. Thus the pattern across the $d^{\prime}$ analysis in this experiment is similar to, but more exaggerated than, that of experiment 6 , showing virtually no effect of polarity for upright faces but near-significant effects of negation with inverted ones.

Analysis of the latencies of correct judgments of sex of head showed significant effects of polarity $\left(F_{1,15}=13.59, \quad p<0.005\right)$ and orientation $\left(F_{1,15}=27.84\right.$, $p<0.001)$ and a significant interaction between sex of head and orientation

(5) As all past research shows negative images to be harder to identify than positive images of faces, we could be justified in using one-tailed tests to examine effects of negation, though we have taken the conservative line of reporting two-tailed significance levels. 
$\left(F_{1,15}=10.97, p<0.01\right)$. These patterns were further complicated by the significant three-way interaction term $\left(F_{1,15}=7.16, p<0.05\right)$. Analysis of this three-way interaction reveals significant and independent effects of polarity and orientation for the female heads, but an interaction between orientation and polarity for the male heads. For the male heads, the effect of negation does not quite reach significance for the upright heads but does for the inverted heads. Overall, however, this three-way interaction seems to reflect different sizes of negation effect for some cells in the design, rather than the absence of an effect in any. A more straightforward picture is observed if analysis of latency is repeated pooling across male and female faces. In this case analysis of variance reveals significant and additive effects of orientation $\left(F_{1,15}=27.8, p<0.001\right)$ and polarity $\left(F_{1,15}=13.6, p<0.005\right)$, and no interaction $(p>0.25)$ between these two factors.

Table 7. (a) Mean percent correct responses and correct-decision latencies (in milliseconds) (shown in parentheses) in experiment 7.

\begin{tabular}{lll}
\hline Orientation & \multicolumn{2}{l}{ Image } \\
\cline { 2 - 3 } & positive & negative \\
\hline Male faces & & \\
Upright & $94.7(870)$ & $95.9(918)$ \\
Inverted & $76.3(1052)$ & $66.1(1250)$ \\
Female faces & & \\
Upright & $84.8(871)$ & $86.0(984)$ \\
Inverted & $80.9(1020)$ & $78.9(1080)$ \\
\hline
\end{tabular}

(b) Mean $d^{\prime}$ scores obtained by combining correct "male" responses with incorrect "male" responses.

\begin{tabular}{lll}
\hline Orientation & Image & \\
\cline { 2 - 3 } & positive & negative \\
\hline Upright & 3.2 & 3.2 \\
Inverted & 2.0 & 1.3 \\
\hline
\end{tabular}

\subsection{Discussion}

The results of experiment 7 replicate and clarify those of experiment 6 showing significant effects of negation, which are (roughly) independent of the effects of orientation, on response latencies in this sex-classification task. The effects of negation on accuracy were slightly stronger in this experiment, where there was a marginally significant trend towards an effect of negation for inverted faces only. The demonstration of significant effects of negation by the use of heads containing no pigmentation cues strengthens the claim that negation affects the computation of 3-D shape from shading, and is disrupted by reversing the pattern of brightnesses from that which would normally be seen. A negative image of a top-lit face presents a pattern of shading which is not the same as that which would arise from moving the lighting direction, since negation inverts the lighting pattern in two dimensions rather than in one. The suggestion that $3-\mathrm{D}$ shape provides useful information for sex judgment is consistent with other data presented by Bruce et al (1993) and Burton et al (1993). However, whereas inversion effects have shown up reliably in this and all earlier experiments, in terms of accuracy, the same is not true of negation where clear effects emerge only on latency. We explore this in discussing the results of all our experiments in section 10 below. 


\section{General discussion}

To recapitulate, in experiment 1 in this paper we observed independent effects of negation and inversion in a task requiring access to face identities. These effects add to those previously observed in tasks of locating face features (Kemp et al 1990) and judging the sex of faces (Bruce et al 1993).

However, in experiments 2 to 4, we showed that when the images of faces that were to be identified lacked pigmentation, effects of inversion remained but those of negation were dramatically reduced, compared with those seen in experiment 1 . Where the effect of negation appeared clearest (experiment 4) it appeared to arise from a response bias rather than difficulty with discrimination. The absence of clear effects of negation in these latter experiments does not seem to arise simply as a result of the different task demands from experiment 1 , since when an experiment with a design the same as experiment 4 was conducted on normal photographs of faces, the standard pattern of inversion and negation was again observed.

When the task to be performed with the nonpigmented facial surfaces was shifted to sex discrimination, in experiments 6 and 7, effects of negation were observed, but these showed up in the latency, rather than in the accuracy, of responding. This suggests that the 'impossible' lighting which is presented by a negative image makes it difficult (and hence more time consuming), but not impossible, to retrieve information needed to make accurate sex judgments.

In contrast, however, effects of inversion showed up reliably on accuracies in all experiments reported here. The size of the effects observed with the nonpigmented laser heads is, if anything, greater than with photographs of faces. For example, in experiments 2,3 , and 4 inversion reduced accuracy of identification by an average of $20 \%, 17 \%$, and $24 \%$, respectively, compared with a reduction of $9 \%$ in experiment 5 in which photographs of faces were used in a task with the same chance rate. In experiments 6 and 7 inversion reduced the accuracy of sex judgments by $11 \%$ and $13 \%$, respectively, compared with an effect of $15 \%$ reduction in accuracy of judging the sex of photographs of faces found by Bruce et al (1993). Effects of negation, where found with the laser heads, were in terms of slowing of responses in sex judgments, though there are nonsignificant trends in the accuracy data in most of the experiments. The nonsignificant effects of negation on identification were of the order of $4 \%$ in experiments 2 and 3 compared with $14 \%$ in experiment 5 in which photographs of faces were used, and on sex judgment were about $3 \%$ in experiments 6 and 7 compared with $11 \%$ obtained with face photographs by Bruce et al (1993). Thus, while we would not want to conclude that negating the laser images had no effect on accuracy of identification and sex judgment, the effects are much reduced compared with those found in equivalent experiments using photographs.

The absence of significant effects of negation on accuracies suggests that reversal of pigmentation is an important component of the effects of negation across all tasks, and indeed appears to be largely responsible for the effects of negation in identification tasks. Clearly, for identification tasks, the presentation of a brunette as an apparent blonde is misleading, and performance suffers as a result. Performance does not suffer, however, from presenting the surfaces with impossible patterns of shading, though this does tend to bias subjects to think that the heads presented in unfamiliar shading patterns are actually unfamiliar.

The surface images of heads derived from laser scanning differ from photographs not just through lack of pigmentation, but also through altered shape cues. Is it the pigmentation or the shape of the hairstyle and eyes which gives rise to the effect of negation? We cannot answer that conclusively for the identification tasks. However we do note that when identification of surface images of faces is compared with the identification of photographs of the same individuals with hair concealed with a 
swimming cap and eyes closed, the surface images are extremely difficult to recognise compared with the photographs (Bruce et al 1991), and this is particularly true for female faces. This effect cannot be due to the missing shape of hair and eyes in the surface images, since it is missing also in the comparison photograph condition, and these results also point to the important role played by pigmentation in identification. Moreover, the additive effects of inversion and negation previously found for judging the sex of faces (Bruce et al 1993) were found when images with hair concealed and eyes closed were used. Overall then it seems that it is the presence, absence, or inversion of pigmentation, rather than particular shape clues, that seems to determine effects in these tasks.

Our results thus provide rather little support for the use of shape from shading in providing information important for face identification. This observation is relevant for our interpretation of previously published data on the identification of line drawings. Davies et al (1978) found that line drawings of famous faces were very poorly identified compared with the original images of these faces, even when the line drawings outlined superficial aspects of the face, such as wrinkles, as well as the major features such as the eyes. In a later paper, Bruce et al (1992) demonstrated that line drawings which preserved 'mass', where dark parts of the original image were portrayed as black in the drawing, were identified almost as well as the original images. This suggested that drawings of faces must preserve some information about the pigmentation and/or shading of the face to be well identified. The results presented here suggest that the effect of mass is a result of preserving information about relative pigmentation rather than shading.

It would appear from our results that reversal of pigmentation also has some effect on sex-classification tasks as well, because negating the surface images did not much affect the accuracy of sex classification, even though it is strictly irrelevant for the performance of this task. Perhaps the unfamiliarity of images of faces in which, for example, eyebrows are lighter than skin, may interfere with normal facial image processing? One obviously novel aspect of a negative image of a face is that the pupils are light rather than dark against dark 'whites' of eyes. To the extent that the eyes provide the frame of reference for face processing, such novelty might be expected to disrupt routines, and might explain why negation, but not inversion, effects were still observed in Kemp et al's (1990) study when face features were replaced by black circles. However, it is important to note here that in Bruce et al's study with negated photographs in a sex-classification task, all the faces were pictured with their eyes closed, yet significant effects of negation on accuracy were still observed. It is nevertheless possible that reversing the pigmentation values of textured regions such as eyebrows on skin may make it harder to determine simple dimensions such as the thickness, bushiness, or setting of brows, all of which provide information which is both diagnostic and used in sex discrimination (Bruce et al 1993; Burton et al 1993). However, the results are not inconsistent with some use of shading information for sex classification since effects on latency of responding were observed. It appears, however, that negative images make it difficult rather than impossible to derive certain information useful for this task.

Acknowledgements. This research was supported by a grant to Vicki Bruce and Mike Burton at the University of Nottingham from the Science and Engineering Research Council's Image Interpretation Initiative (GR/F 33698). The work was conducted in collaboration with Alf Linney and Anne Coombes (University College, London), who, along with Mike Burton and Elias Hanna at Nottingham, made essential contributions to this research by producing the surface images used in the experiments. Alan Milne at the University of Wales wrote the tachistoscopic software for the MacIIcx. Experiment 5 was suggested by a reviewer of an earlier version of the manuscript. 


\section{References}

Bartlett J C, Searcy J, 1993 "Inversion and configuration of faces" Cognitive Psychology 25 $281-316$

Bruce V, 1988 Recognising Faces (London: Lawrence Erlbaum Associates)

Bruce V, Burton A M, Hanna E, Healey P, Mason O, Coombes A, Fright R, Linney A, 1993 "Sex discrimination: How do we tell the difference between male and female faces" Perception 22 131-152

Bruce V, Hanna E, Dench N, Healey P, Burton M, 1992 "The importance of "mass" in linedrawings of faces" Applied Cognitive Psychology 6 619-628

Bruce V, Healey P, Burton M, Doyle T, Coombes A, Linney A, 1991 "Recognising facial surfaces" Perception $20755-769$

Bruce V, Young A W, 1986 "Understanding face recognition" British Journal of Psychology 77 $305-327$

Burton A M, Bruce V, Dench N, 1993 "What's the difference between men and women?" Perception 22 153-176

Davies G M, Ellis H D, Shepherd J W, 1978 "Face recognition accuracy as a function of mode of representation" Journal of Applied Psychology 63 180-187

Diamond R, Carey S, 1986 "Why faces are and are not special: An effect of expertise" Journal of Experimental Psychology: General 115 107-117

Galper RE, 1970 "Recognition of faces in photographic negative" Psychonomic Science 19 207-208

Galper RE, Hochberg J, 1971 "Recognition memory for photographs of faces" American Journal of Psychology $84351-354$

Goldstein A G, Chance J E, 1981 "Laboratory studies of face recognition", in Perceiving and Remembering Faces Eds G Davies, H Ellis, J Shepherd (London: Academic Press)

Hayes A, 1988 "Identification of two-tone images: some implications for high- and low-spatialfrequency processes in human vision" Perception 17 429-436

Hayes T, Morrone M C, Burr D C, 1986 "Recognition of positive and negative bandpassfiltered images" Perception 15 595-602

Johnston A, Hill H, Carman N, 1992 "Recognising faces: effects of lighting direction, inversion, and brightness reversal" Perception $21365-375$

Kemp R, McManus I C, Pigott T, 1990 "Sensitivity to the displacement of facial features in negative and inverted images" Perception $19531-543$

Linney A D, 1992 "The use of 3-D computer graphics for the simulation and prediction of facial surgery", in Processing Images of Faces Eds V Bruce, M Burton (Norwood, NJ: Ablex) pp 149-178

Phillips R J, 1972 "Why are faces hard to recognise in photographic negative?" Perception \& Psychophysics 12 425-426

Rhodes G, Brake S, Atkinson A P, 1993 "What's lost in inverted faces?" Cognition 47 25-57

Roberts T, Bruce V, 1988 "Feature saliency in judging the sex and familiarity of faces" Perception $17475-481$

Scapinello F F, Yarmey A D, 1970 "The role of familiarity and orientation in immediate and delayed recognition of pictorial stimuli" Psychonomic Science 21 329-331

Sergent J, 1984 "An investigation into component and configural processes underlying face recognition" British Journal of Psychology 75221 - 242

Tanaka J W, Farah M J, 1993 "Parts and wholes in face recognition" Quarterly Journal of Experimental Psychology 46A 225-246

Thompson P, 1980 "Margaret Thatcher-a new illusion" Perception 9 483-484

Valentine T, Bruce V, 1988 "Mental rotation of faces" Memory \& Cognition 16 556- 566

Yin R K, 1969 "Looking at upside-down faces" Journal of Experimental Psychology 81 141 - 145

Young AW, Bruce V, 1991 "Perceptual categories and the computation of Grandmother" European Journal of Cognitive Psychology 3 5-49

Young A W, Hellawell D J, Hay D C, 1987 "Configurational information in face perception" Perception $16747-759$ 